ÁMBITOS. № 15 - Año 2006 (pp. 12-156)

\title{
La (lógica) construcción de la realidad
}

\author{
Lázaro Carrillo Guerrero \\ Universidad de Granada \\ Izro@msn.com
}

\section{Resumen:}

Toda actividad de la lengua requiere unas representaciones cognitivas y unas construcciones lógicas para actuar sobre el mundo y situarse ante lo necesariamente o probablemente verdadero. Ello hace que, en la comunicación lingüística, el texto se construya de acuerdo a unas alternativas lógicas particulares y sociales, y que dentro de cada discurso haya una racionalidad construida adaptable a los interlocutores y a las situaciones, y variable de acuerdo con las capacidades de los participantes y sus cálculos estratégicos. En este marco, de interacción social y de relación lengua-pensamiento, se despliega una lógica argumentativa que se caracteriza por ser abierta a la situación comunicativa, y por estar marcada de forma prominente por un carácter expresivo, convencional, o retórico. De modo que, usar la lengua es interpretar la realidad, trazándola, al menos, desde dos perspectivas: la del hablante y la del oyente. Siendo la construcción retórica del texto la que da forma a la manera en que la gente, en su vida diaria, ve su realidad y su existencia. Se trata de estructuras sintácticas funcionando en una dinámica racional de interacción comunicativa y construyendo unas realidades comunicativas con un determinado sentido lógico y una determinada fuerza pragmática. $Y$ donde se establece una correspondencia entre la estructura del texto y la estructura del mundo, y donde la acción del discurso construye una realidad exterior y sustenta una realidad interna.

\begin{abstract}
:
Every language activity requires some cognitive representations and some logic constructions in order to function upon the world and to get a position in the face of what is necessarily or probably truthful. In linguistic communication, this makes that the text be built according to some concrete and social logic alternatives, and that within each discourse there have an erected reality that is adaptable to the interlocutors and to the situations, and variable according to the participants' capacity and their strategic conjectures. In this frame, of social interaction and of language-thought relation, it is spread out an argumentative logic that is characterized by being opened to the communicative situation, and by being marked in a prominent manner with an expressive, conventional or rhetoric character. Therefore, to use language is to interpret reality, sketching it, at least, from two perspectives: that of the speaker and that of the hearer. Being the text's rhetoric construction what gives form to the manner in which people, every day, see their reality and their existence. It is about syntactic structures functioning in a rational dynamic of communicative interaction and constructing some communicative realities with a certain logic sense and a definite pragmatic force. Where there is established a correlation between the text structure and the world structure, and where the discourse's action builds an exterior reality and supports an interior reality.
\end{abstract}

Palabras clave: Lógica, lengua natural, retórica, argumentación, construcción textual, interacción comunicativa, discurso, realidad comunicativa, cognición.

Key words: Logic, natural language, rhetoric, argumentation, textual construction, communicative interaction, discourse, communicative reality, cognition. 


\section{INTRODUCCIÓN}

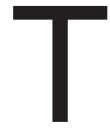

oda codificación lingüística obedece a unos esquemas mentales: cognitivos (representaciones) y lógicos (construcciones). Ambas, representaciones y construcciones, van en función de lo que opera sobre nosotros al escuchar, y lo que opera sobre nosotros al enunciar. Lo cognitivo va a constituir la interiorización de nuestras representaciones de las realidades del mundo, y los medios, las estrategias, por los cuales vamos a proceder a construir estas representaciones. La cuestión está en saber si la realidad que nosotros percibimos (construida con nuestras experiencias individuales y conocimientos colectivos) es la verdadera. La noción de representación es indisociable de aquella de comunicación en el sentido de la acción simbólica sobre el mundo y sobre el otro. Toda actividad de la lengua, en la medida donde ella se constituye como actividad cognitiva de representación de eventos o de objetos del mundo, va a construir un esquema, es decir, una figura reducida de este mundo. Esta esquematización, a la vez cognitiva y lingüística, opera con unas finalidades en cada discurso: orientar al otro hacia un sentido, hacia una cierta concepción del mundo.

\section{LÓGICA FORMAL, LENGUA NATURAL}

Dik (1989:4) define la lengua natural como un instrumento de interacción social, que existe en virtud de ser usada para ciertos propósitos, y teniendo como función la comunicación entre sus usuarios. En contraste con diferentes consideraciones respecto a la lengua, él distingue dos paradigmas:

a) Paradigma formal: la lengua es considerada como un objeto formal y abstracto; y se le da un prioridad metodológica a la sintaxis sobre la semántica y la pragmática.

b) Paradigma funcional: la lengua es conceptualizada como un instrumento de interacción social, con la intención de establecer unas relaciones comunicativas; y la pragmática es vista como un marco dentro del cual se sitúan la semántica y la sintaxis.

Esta distinción nos puede servir para situarnos ante lo que podríamos llamar "la lógica formal en la lengua" (el paradigma formal), y ante "la funcionalidad de la lengua natural" (el paradigma funcional). Es decir, ante lo que Kress (1992:187) Ilama (respectivamente) lo "necesariamente verdadero", y lo "probablemente verdadero".

Dik (1989:1), al plantear un modelo del usuario de la lengua natural, distingue, a través de las expresiones lingüísticas, cinco capacidades esenciales en la comunicación lingüística:

(i) una capacidad lingüística (que produce e interpreta correctamente las expresiones lingüísticas),

(ii) una capacidad epistémica (que construye, mantiene y explota una base de conocimiento organizada), 
(iii) una capacidad lógica (que deriva posteriores piezas de conocimiento por medio de reglas de razonamiento, que son movidas por principios de lógica deductiva y probabilística),

(iv) una capacidad perceptiva (que percibe su ambiente y deriva conocimiento de sus percepciones),

(v) una capacidad social (que hace conocer qué decir y cómo decirlo, para conseguir unos objetivos comunicativos)

Esto nos lleva a considerar que, dentro de cada discurso hay una racionalidad construida, a través de sus estructuras, y a través de un contenido que es lógicamente coherente. La referencia del texto obedece a alternativas lógicas particulares y sociales. $\mathrm{Y}$ siempre, en nuestro uso de la lengua, realizamos procesos de razonamiento. Esta racionalidad es adaptable a los interlocutores y a las situaciones, y es variable de acuerdo con las capacidades de los participantes en la comunicación y de acuerdo con los cálculos estratégicos. Por ejemplo, el texto siguiente, extraído de una secuencia de la película Wonder Boys :

[Grady llama por teléfono a Sara, desde la casa de los padres de Emily, su esposa. Dónde él y James han entrado, estando los padres de Emily fuera de la casa, y no habiendo nadie en ella]

GRADY: (en el teléfono, y hablando con otra persona) Yes, l'm looking for the Chancellor... I don't know. She should be in the main hall...Thank you.

[Sara se pone al teléfono]

GRADY: Sara? Hi. It's Grady.

SARA'S VOICE: Where are you, Grady? An elevator?

GRADY: I'm in Kinship. Listen, Sara, there's some things we need to talk about...

SARA'S VOICE: (apaciblemente) You're in Kinship? [En Kinship está la casa de los padres de Emily, la esposa de Grady, casa de dónde está llamando Grady]

GRADY: Yes. But that's not why I called...

SARA'S VOICE: With Emily?

GRADY: What? No'. There's no one here. I'm just...just....

SARA'S VOICE: Just what? Doing a little dusting?

[James tiene la televisión en un volumen de sonido alto]

GRADY: (gritando) James! For Christ sake, will you turn that thing down!

\footnotetext{
${ }^{1}$ Dirigida por Curtis Hanson, 2000, Mutual Film Company y Paramount
} 
SARA'S VOICE: James? He's still with you?

[Grady mueve la cabeza y lleva el teléfono hacia la ventana]

GRADY: Look, Sara. ..I'm not here... I'm not here to...reconcile with Emily.

SARA'S VOICE: Are you there to not reconcile with her?

[Grady ve por la ventana a sus suegros bajándose del coche y dirigirse hacia la casa; eso le hace estar a Grady momentáneamente en silencio. Silencio que Sara interpreta a su manera] SARA'S VOICE: Goodbye, Grady.

GRADY: No. Sara, you don't understand...

SARA'S VOICE: Trust me, I understand. I just want to say something to you, Grady. GRADY: (temeroso) Yea?

SARA'S VOICE: How you choose to live your own life is your business. But you be careful with that boy, Grady. With James. He belongs to somebody else.

[La línea se corta, y Grady ve que sus suegros ya han entrado en la casa] nos muestra estructuras tales como:

GRADY: Sara, ..I'm not here... I'm not here... I'm not here to... reconcile with Emily. SARA'S VOICE: Are you there to not reconcile with her?.

donde los dos interlocutores estructuran sus enunciados de acuerdo a unos procesos de razonamiento que obedecen a una lógica particular de cada uno. Grady, conociendo su situación, niega una conclusión que está implícita y es probable, pero no es verdadera. En este acto ilocutivo de Grady hay un razonamiento basado en la conclusión a la que puede llegar su interlocutor, Sara. Y el enunciado interrogativo de Sara, desde su posición (estando al otro lado del teléfono y siendo amante de Grady), tiene el objetivo de llegar, con alto grado de fuerza retórica, a una conclusión que parece lógicamente evidente y probable, pero que tampoco es verdadera. En este acto ilocutivo de Sara hay un razonamiento basado en la evidencia (con carácter general y social) de la situación en la que Grady se encuentra: él esta en la casa de los padres de Emily, su esposa (quién le ha abandonado).

En contraste con la retórica, preocupada por el auditorio ${ }^{2}$, la lógica ${ }^{3}$ siempre ha estado preocupada por demostrar algo como concluyente y cierto, y también por mostrar el carácter razonable o verosímil de una propuesta. Para ambas preocupa-

\footnotetext{
${ }^{2} \mathrm{Y}$ preocupada por lograr una comunicación efectiva.

${ }^{3}$ Perelman (1983) aboga por una conciliación entre la razón analítica y la razón dialéctica. Él asigna el campo de las ciencias matemáticas y físicas a la lógica (la razón analítica), y el campo del humanismo a la retórica (la razón dialéctica). De esta forma, Perelman contrasta un sistema filosófico "regresivo", donde el énfasis está en la argumentación, es decir, en la dialéctica y la retórica, con un sistema filosófico "primario", donde el énfasis está en la demostración, en la lógica y analítica.
} 
ciones necesitamos utilizar las estructuras de la lengua. Unas estructuras que llevan consigo un conocimiento y un significado, siendo ambos muy sensibles a los contextos en que son usados. Así, en las actitudes del hablante y del oyente hay una lógica determinada que interviene en todo tipo de discurso. Por ejemplo:

a) la conexión lógica de ideas y conceptos, a través de las estructuras lingüísticas, y a través de la conexión temporal de acciones y hechos, o a través de una interacción de intenciones y acciones, de acuerdo con una situación;

b) el uso de los conectores lógicos ${ }^{4}$ (therefore, well, so, thus, as a result, consequently, etc.), que contribuyen a la coherencia lógica del discurso; la integración ${ }^{5}$ de estructuras y significados no expresados directamente en el discurso; etc.

El ejemplo anterior, del texto extraído de la película Wonder Boys, presenta una lógica a través de: una interacción de intenciones, por parte de ambos interlocutores, Grady y Sara; y de la integración de estructuras y significados implícitos, como es la conclusión que Grady quiere evitar, y a la que Sara quiere llegar. Además, en el texto podemos encontrar la conjunción coordinante but contribuyendo a una coherencia lógica entre significados y estructuras:

- SARA'S VOICE: You're in Kinship? / GRADY: Yes. But that's not why I called...

- SARA'S VOICE: How you choose to live your own life is your business. But you be careful with that boy. ...

Aunque, siguiendo la línea tradicional, se podría emparejar la razón analítica con la lógica formal ${ }^{6}$, y la dialéctica con el lenguaje natural, entendemos que la lengua común, el uso de la lengua natural también tiene una lógica. Una lógica fundada sobre la noción de probabilidad, y que Toulmin (1983) llama lógica substantiva (substantive logic). Es decir, en el uso cotidiano de la lengua, no se realiza un razonamiento formal, basado en una certeza matemática, sino que utilizamos un razonamiento no formal, basado en la probabilidad. Pero, de acuerdo con Grize (1982:173), la lógica natural es irreducible a la lógica matemática, aunque compatible con ella. Grize (1982) afirma que la lógica natural opera a través de unas esquematizaciones probables por medio de la palabra. Él define la noción de esquematización, de una forma metafórica, como "la elaboración, por medio de la lengua, de un micro-universo que A presenta a B con la intención de obtener un cierto efecto sobre él” (Grize, 1982:188). Se trata de la elaboración del discurso

\footnotetext{
${ }^{4}$ Los lógicos, en sus teorías, operan con unos operadores (o signos) lógicos que sustituyen a algunas palabras en la lengua, tales como: and (conjunction), or (disjunction).

${ }^{5}$ Ayudado por lo que Kress (1992:177) llama, script-based knowledge; y por la situación.

${ }^{6}$ Este tipo de lógica podría definirse, de acuerdo con Plantin (1990:170), como la sistemática de una estructura reglada, coherente, capaz de auto-reproducirse y de funcionar auto-referencialmente, libre de toda coacción contextual.
} 
argumentativo en la interacción comunicativa ${ }^{7}$. Y entendemos que esta esquematización supone la práctica del discurso y de la retórica para organizar una argumentación en función del interlocutor y de las finalidades y objetivos que se pretenden. El texto analizado anteriormente muestra como Sara presenta, mediante su pregunta: You're in Kinship?, un esquema argumentativo orientado hacia una conclusión (You're there to reconcile with Emily), todo ello de forma implícita. Y Grady, mediante su declaración afirmativa/negativa: Yes. But that's not why I called..., muestra compartir cognitivamente ese esquema, pero presentándolo a Sara como no aplicable a su situación.

Se trata de una lógica que no está basada en premisas verdaderas y explícitas, e inferencias concluyentes, sino en principios retóricos, que no necesitan ser explicitados, pero que son plausibles. El uso del lenguaje natural no se caracteriza por unos argumentos lógico-matemáticos, sino por una inestabilidad semántica, por una ambigüedad en la conducta lingüística, por un significado dirigido y condicionado por el cotexto y por su contextualización. Se trata, pues, de una lógica abierta adaptable a la situación comunicativa.

La lógica formal y el razonamiento silogístico tienen un campo limitado: el pensamiento racional puramente formal, la lengua escrita, y un uso descontextualizado de la lengua. Aunque, estimamos, con Leith y Myerson (1989), que, también, tiene un determinado tipo de contexto:

Syllogisms, for instance, are part of a language-game, associated with formal education, of 'Let's assume that ...' and participants have to know the rules of that particular game if they are to produce acceptable moves (Leith y Myerson, 1989:15).

Pero su preocupación por la verdad y falsedad de las proposiciones no participa de la otra lógica, la lógica de la situación y de la fuerza comunicativa ${ }^{8}$. En la lógica de la lengua natural, al ejecutarse siempre en una situación comunicativa, disponemos de distintas alternativas de mirar al mundo, y la posibilidad de no ser atrapados por un sistema dominante de creencias.

O'Keefe (1986; en Willard, 1991:99-102) distingue tres lógicas diferentes, a través del uso de los conceptos que constituyen la comunicación, y que producen diversos modelos de organización del mensaje y de interpretación:

\footnotetext{
${ }^{7}$ En este marco de la interacción comunicativa, la lengua despliega una dimensión argumentativa, ya que su uso tiene un rol intencionado, y éste existe de acuerdo con las relaciones que se establecen entre los interlocutores.

8 "J.L. Austin's "How to do things with words" was a milestone because it offered to release linguistic philosophy from the age-long tyranny of its preoccupation with the truth and falsehood of propositions. The chief instrument of this liberation was the performative sentence, which, declarative in form, seemed to call for a logic quite different from that of normal declarative sentences: a logic of performance and of felicity conditions, rather than of statement and of truth conditions" (Leech, 1980:79).
} 
a) La lógica del proyecto expresivo, que se basa en la noción de que la lengua es un medio para expresar los pensamientos y los sentimientos, donde, la única tarea que puede realizar un mensaje es la expresión. El mensaje es interpretado como una unidad independiente, más bien que como un hilo dentro de un tejido hecho de interacciones.

b) La lógica del proyecto convencional, que se basa en la noción de que la comunicación es un juego cooperativo jugado según unas reglas convencionales y unos procedimientos fijados socialmente. Esta lógica asume que la lengua es un tesoro de producciones expresivas; pero las producciones que se expresan son determinadas por el resultado social que se les quiere dar. Así, mientras que los mensajes expresivos son, más bien, psicológicos y reactivos en sus relaciones con el contexto, los mensajes convencionales comportan una relación convencionalizada y reglada con el contexto.

c) La lógica del proyecto retórico, que se basa en la noción de que la comunicación es creación y negociación de los individuos y de las situaciones sociales. En esta lógica, el conocimiento convencional se asume bajo la visión de unos individuos y unas situaciones móviles, más bien que estables, donde se realizan unas negociaciones sociales y se muestra una realidad social particular. La función de los mensajes retóricos es la negociación entre interlocutores para llegar al consenso.

Estas tres lógicas, aunque diferentes, nosotros las entendemos como incrustadas una dentro de otra, y las tres con una proyección retórica. Es decir, las reglas de: tener tacto, comunicar con franqueza (proyecto expresivo), ser cortés, usando formas corteses convencionales (proyecto convencional), y considerar al otro y a las situaciones (proyecto retórico), son reglas retóricas, que en una relación lógica entre ellas quedarían una abarcada y controlada, de alguna forma, por la otra, y de la siguiente manera:

\section{\{\{\{Lógica expresiva\} Lógica convencional\} Lógica retórica\}}

Y cada una de ellas puede ser prominente en la acción de una lógica argumentativa ${ }^{9}$, y marcar a esta acción por su carácter lógico-expresivo, lógico-convencional, o lógico-retórico. Por ejemplo, el texto extraído de la película Wonder Boys muestra como, en su conjunto, desarrolla un lógica retórica, ya que está basado en la interacción y negociación de significados entre los interlocutores. Pero, por debajo de ella hay una lógica convencional, mayormente influenciada por "modo" y "campo", y que caracteriza unos diálogos cortos en un modo interrogativo y declarativo. Y, dentro de esta convencionalidad, las estructuras de significado que

\footnotetext{
${ }^{9}$ O lógica discursiva que, en un mundo referencial y comunicativo, construye unas relaciones lógico-semánticas en el texto y procesa unas estructuras lingüísticas concluyentes.
} 
se utilizan, reflejan cada una unos sentimientos y pensamientos. Sin embargo, el siguiente texto publicitario ${ }^{10}$ muestra (dentro de la incrustación de las tres lógicas) una cierta prominencia en su acción lógico-convencional:

LOOK GOOD. FEEL BETTER. BE YOUR BEST. No matter what your body type, this is the best workout video for you.

Donde tanto el mensaje que transmite:

- el logro de un bienestar físico y social a través de un video,

como las formas que utiliza:

- la iconicidad de letras mayúsculas (formando oraciones, en modo imperativo, muy simples y breves, fáciles de captar y entender a primera vista, y sugiriendo bienestar) en contraste con las minúsculas (formado oraciones simples, que en modo declarativo incitan a comprar). Y marcando con ello el foco informativo y la prominencia comunicativa en la primera parte del mensaje, están determinadas por los procedimientos comunicativos y los resultados a obtener que mueven al discurso publicitario.

Las reglas lógicas adaptadas al flujo de intercambios lingüísticos reposan sobre una base que es retórica: lo probable, la comunicación de ideas, la intencionalidad y efectividad comunicativas... La lengua ordinaria, la que usamos todos los días, y el razonamiento basado en el sentido común, la lógica de todos los días, no se adhieren estrictamente a los procesos lógicos condicionados por valores de verdad, sino que más bien los evitan. De esta forma, hacemos inferencias, y llegamos a conclusiones, que son incitadas por el propio proceso discursivo en el que actuamos, pero que posiblemente no sean necesariamente correctas desde un punto de vista estrictamente lógico (Meijs, 1992). Muy frecuentemente actuamos intuitivamente de una forma lógica, sin detenernos a analizar nuestros propios procesos lógicos (Kinneavy, 1971:106). Pero la versatilidad de la lengua requiere también de esas intuiciones lógicas que ella misma nos proporciona.

El razonamiento por inferencia, la deducción, las demostraciones, etc., todos formando parte del modelo mental del discurso, requieren del uso de la lengua y del uso de la lógica ${ }^{11}$ en las interacciones comunicativas. Interacciones, donde la relación dinámica entre lengua y pensamiento puede ser constante o variar sistemáticamente:

The thought-language relationship is one that exists not only, or even primarily, in the heads of participants, within their linguistic systems or vocabularies, nor merely as the 'folk psychology' they use, but it is dealt with by participants as a practical matter, a concern of theirs that they handle in talk and text (Edwards, 1997:10).

\footnotetext{
${ }^{10}$ Insertado en una página de una revista, y junto a la imagen de una top model (Elle Macpherson), funciona como publicidad para la venta de un video titulado: "Your Personal Best" para hacer ejercicios.

${ }^{11}$ La lógica no formal, en cierto modo, ha representado una insatisfacción hacia la lógica deductiva formal.
} 
y donde constantemente estamos haciendo inferencias y deducciones mediante una relación lengua-pensamiento que se desarrolla en una lógica probabilística, y en el marco de las situaciones comunicativas.

Anscombre (1991:140) contrasta, en la lengua, el enfoque veri-condicional y el enfoque argumentativo. Ambos tendrán en común parte de la base de datos, y el conocimiento del mundo exterior. Pero la parte propiamente lingüística será un conjunto de constantes léxicas en el enfoque veri-condicional, y un conjunto de topois (principios generales o lugares comunes) en el enfoque argumentativo. En el nivel de la inferencia, el enfoque veri-condicional utiliza una deducción fundamentada en unas implicaciones generales o locales. En contraste, el enfoque argumentativo utiliza un modo de inferencia escalar (en escala). Ello hace que el razonamiento en la lengua tenga más de probable que de verdadero, más de apuesta o de exageración que de cuantificación. Si hay una lógica de la lengua a inventar, ella resultará de lo probable, y no de la cuantificación.

\section{CONCEPCIÓN DEL MUNDO}

Nuestro conocimiento del mundo se procesa, se recibe, y se almacena en estructuras lingüísticas. Así un texto refleja la percepción de la realidad de los hablantes, y sus estructuras constituyen el significado que estos les asignan ${ }^{12}$. El mundo social se construye textualmente en una interacción que depende de varios factores contextuales, pero principalmente de quienes lo están construyendo (ver Sayer, 2000, sobre "construccionismo social"). Y usar la lengua es construir la forma que sus usuarios requieren. Pero, esta construcción textual es variable, tanto en el individuo como en la comunidad lingüística, y de acuerdo con las interacciones de quienes construyen el texto. De modo que, usar la lengua es interpretar la realidad. Pero trazándola, al menos, desde dos perspectivas: la del hablante y la del oyente. Aunque, podría haber otras perspectivas en la interacción textual, si consideramos que pueden existir otras voces y otros auditorios implicados en esa interacción. Pero lo más complejo y a la vez más interesante, es que siempre existe, en estas perspectivas sobre la realidad, una intención de actuar sobre las otras perspectivas (intentando alterarlas de alguna forma). Y entendemos que en esa actuación se construye una realidad, o la realidad. Así, en el texto extraído de la película Wonder Boys, Grady y Sara nos muestran las realidades que construyen (las flechas las señalan), cada uno, en su interacción y de forma implícita, para actuar, recíprocamente, sobre el otro:

GRADY: Sara, ..I'm not here... I'm not here... I'm not here to...reconcile with Emily. ? [You think I'm here to reconcile with Emile, and I'm not]

\footnotetext{
${ }^{12}$ Según Sapir y Whorf (Whorf, 1956), cada lengua tiene una estructura que debe de algún modo influenciar la manera en que sus hablantes ven el mundo. Ellos investigaron la relación entre lengua y pensamiento.
} 
SARA'S VOICE: Are you there to not reconcile with her?.? [Being there, in Kinship, has only one option: to reconcile with Emily]

Searle (1995) defiende la idea de que hay una realidad totalmente independiente de nosotros. Para ello, él distingue (capítulo 1) entre dos tipos de rasgos ${ }^{13}$ : aquellos que son intrínsecos a la naturaleza de la realidad física, y aquellos otros que son intrínsecos a la intencionalidad ${ }^{14}$, opinión, punto de vista, evaluación, y ciertas actitudes y sentimientos de los observadores y usuarios. Este último rasgo conduce, de acuerdo con Searle, a la funcionalidad, donde las funciones, no son nunca intrínsecas, sino que se asignan a la realidad de forma relativa, y de acuerdo a los intereses de los observadores y usuarios. Con respecto a las funciones, Searle distingue entre agentive y nonagentive functions. Mientras que las últimas son procesos naturales (por ejemplo, el bombeo del corazón), las primeras son procesos de simbolización y de representación para significar algo. Y es aquí, donde nosotros entendemos que la lengua da peso y hace visible una realidad, que Searle (1995:4) define así: "...the complex structure of social reality is, so to speak, weightless and invisible." En esta actuación sobre la realidad, Searle (1995:23-26) distingue entre una intencionalidad colectiva (We intend, We consciousness), donde nosotros vemos un cierto paralelismo con el concepto de "Comunidad Discursiva" ${ }^{15}$; y una intencionalidad individual (I intend, I consciousnesses) de cada persona, derivada de la intencionalidad colectiva que comparte en su comunidad. Así pues, para esta funcionalidad y para esta intencionalidad se requiere la lengua, considerada por Searle como una institución. Él (1995:59) la califica de: “...essentially constitutive of institucional reality." Es decir, la funcionalidad, la intencionalidad, y la realidad institucional necesitan de la lengua. Necesitan de recursos simbólicos tales como las palabras (además de otros recursos convencionales). Y esta necesidad de la lengua con respecto a la realidad es doble:

1) necesidad de representar lingüísticamente a la realidad,

2) y necesidad de comunicar la realidad mediante el uso de sus estructuras.

Para Searle (1995:90 y ss.), la realidad institucional y social contiene, como elementos constitutivos, a las representaciones mentales y representaciones lin-

\footnotetext{
${ }^{13}$ Searle distingue entre institutional facts y brute (noninstitutional) facts. Los primeros requieren de las instituciones humanas para su existencia. Los segundos requieren de la institución del lenguaje para su existencia.

${ }^{14}$ "Intensionality-with-an-s should not be confused with intentionality-with-a-t. Intentionality is that property of the mind by which it is directed at objects and states of affairs in the world. Intensionality is that property of sentences and other representations by which they fail certain test for extensionality. One of the most famous of these is Leibniz's Law: If two expressions refer to the same object they can be substituted for each other in a sentence without changing the truth value of the sentence. Sentences that fail this test are said to be intensional with respect to substitutability. Another expression used to name this sort of in tensionality is "referential opacity." Typically sentences that are about intentional-with-a-t states are intensional-with-an-s sentences, because in such sentences the way in which an object is referred to affects the truth value of the sentence. For extensive discussion of these matters see Searle Intentionality Art Essay in the Philosophy of Mind" (Searle, 1995:18-19).
} 
güísticas. Ambas tienen unas estructuras lógicas. Y éstas funcionan de acuerdo con unas estructuras de relaciones de poder, donde se incluyen los poderes: negativo y positivo, condicional y categórico, colectivo e individual. Aquí, la estructura lógica básica puede hacerse visible en: We accept ( $S$ has power ( $S$ does $A$ )). Todo ello se realiza en una simbolización que crea lenguaje y significado en todas sus formas para representar una realidad, con posibles modos ilocutivos, a través de unas estructuras de sintaxis y de semántica (de significado).

Searle (1995:150-1) distingue seis rasgos en la concepción del mundo o la realidad, que nos permitimos reducir a dos:

1) Un realismo externo, mediante el cual, el mundo (el universo, la realidad) existe independientemente de nuestras representaciones de él o de ella.

2) Las representaciones (individuales y colectivas) que tenemos y hacemos para acceder a la realidad mediante el pensamiento, las creencias, los deseos, etc., y que realizamos, arbitrariamente, a través de un sistema de representación, tal como es la lengua, la imagen, etc., e influenciados por factores culturales, económicos, sicológicos, etc.

Además, se crean, también unos modos de decir y unas maneras de expresar la realidad física o social. A estos modos y maneras se les puede calificar de socioculturales dentro de una determinada comunidad, y de convencionales dentro del discurso. Y su carácter retórico tiene una función regulativa, que puede llegar a ser manipuladora. Un ejemplo de ello es el texto publicitario anterior, el cual muestra unas formas y unos modos convencionales que pertenecen al discurso publicitario; y presenta una realidad de acuerdo a unos intereses socioculturales creados: LOOK GOOD. FEEL BETTER. BE YOUR BEST. No matter what your body type, this is the best workout video for you.

Desde esta perspectiva, reiteramos que la lengua no solamente construye la realidad, sino que, además, ayuda a crear y refleja los intereses y las ideas de los grupos sociales dominantes. De modo que, a través de la lengua:

a) se puede crear, modificar, y desarrollar un sentido del mundo,

b) se puede institucionalizar lo que es hablado frecuentemente,

c) y se puede debilitar o percibir como inaceptable otras versiones en el uso de la lengua (que pueden o no ser omitidas).

Halliday (1978), desde su consideración de la lengua como una semiótica social, sitúa este poder de la lengua en la interacción:

But, as Berger and Luckmann rightly point out, the reality-generating power of language lies in conversation; furthermore it is cumulative, and depends for its effectiveness on continuous reinforcement in interaction (Halliday, 1978:177) ${ }^{16}$.

\footnotetext{
${ }^{15}$ Esta noción, de acuerdo con los etnógrafos de la comunicación, la entendemos como un grupo de gente que comparten las mismas reglas y modelos en el qué decir, cuándo, y cómo decirlo.
} 
Para Halliday (1978:191), la realidad, siendo una construcción social, solamente puede ser construida a través de un intercambio de significados. Así, la lengua, mientras que representa la realidad referencialmente, a través de sus palabras y estructuras, también representa la realidad metafóricamente, a través de su propia forma interna y externa. De modo que, en el mismo proceso en el que la lengua llega a ser una metáfora de la realidad, la realidad llega a ser una metáfora de la lengua. Con todo ello, Halliday expresa una doble simbolización:

(1) The functional organization of the semantics symbolizes the structure of human interaction (the semiotics of social contexts, as we expressed it earlier). (2) Dialectal and 'diatypic' (register) variation symbolize respectively the structure of society and the structure of human knowledge (Halliday, 1978:191).

Los diferentes cambios de registros lingüísticos nos muestran la relatividad con la que se representa y simboliza la realidad (Fowler, 1996:212), y entendemos que también nos muestran como la retórica, a través de la lengua, da forma a la manera en que la gente, en su vida diaria, ve su realidad y su existencia (ver Owen, 1997).

En Bazerman y Paradis (1991), dentro del constructivismo social a través de los textos escritos, se analizan la importancia que estos tienen en la construcción de las profesiones. Se muestran como las definiciones y las formas textuales imponen su estructura en la actividad humana y ayudan a formar versiones de la realidad. De modo que los textos tienen un rol poderoso en organizar las acciones diarias de los individuos. Y Fairclough (1992, 1995, 2001) examina en el discurso la lucha hegemónica por el poder para construir una realidad social mediante el control intertextual de prácticas discursivas.

La teoría dramatista de Burke (1969) dota a los símbolos, y a su proceso de simbolización, de una función central en la creación y estructuración de la experiencia humana y en la intervención social, y en definitiva en la ideología; e inspira a otros autores en este planteamiento.

Bormann (1985) plantea la idea de una visión retórica común, mediante la cual una comunidad se apropia de una serie de temas y de esquemas y los unifica para producir una realidad simbólica común. A menudo, este proceso de coalescencia se efectúa alrededor de una analogía modelo expresada por una etiqueta o un eslogan, tal como "la nueva política", "la guerra fría", "la mayoría silenciosa", "la nueva derecha", etc.

\footnotetext{
${ }^{16}$ Berger y Luckmann (1976) consideran la conversación como la interacción primaria, mediante la cual se transmite la visión comunitaria del mundo.
} 
Junto con Bormann, McGee $_{17}(1980)$, y Brown (1978), autores y analistas del movimiento retórico epistémico ${ }^{17}$, y desde una perspectiva ideológica, ven en los símbolos el instrumento que permite construir una realidad social, y sitúan la función simbólica en la realidad humana.

McGee (1980) propone el ideógrafo (the ideograph) como una unión entre retórica e ideología. Éste, funcionando vertical u horizontalmente, captura las fuerzas y las intenciones que actúan en una situación retórica, tal como lo testimonian los eslóganes característicos o los ideografos, los cuales constituyen los pilares de la ideología: "propiedad", "religión", "protección de la vida privada", "justicia", "libertad", "libertad de expresión", "igualdad", "orden", etc.

Para Brown (1978) las construcciones simbólicas, que la persona captura de la ideas, ordenan el mundo y le hacen entrar en relación con los otros, dándole a ellos y a él una identidad. De esta forma, una ideología constituye una visión del mundo: los individuos utilizan los símbolos para formar su ideología. Y ésta, la ideología, se manifiesta por una polarización de la atención, que puede variar de acuerdo con los intereses, las intervenciones sociales, y las relaciones de poder. La visión holográfica del argumento de Brown (1978) se basa en esta teoría de la ideología y la intervención social, y sobre todo en los fenómenos de poder.

\section{CONSTRUCCIÓN DE LA REALIDAD}

Beale (1987:66-7) define la construcción de la realidad a través de dos grandes ejes, o dos conjuntos de decisiones que se hacen bien conscientemente, como un asunto de principio, o bien inconscientemente, como un asunto de suposición:

1) Podemos considerar la realidad: a) como algo exterior (out here), existiendo independientemente de la consciencia (se trata de un objetivismo y realismo estricto) y aislada del observador, b) como algo interior (in here), que incluye la consciencia o que es primariamente un asunto de consciencia (se trata de un relativismo y subjetivismo estricto) en interacción con el observador, c) o como algo entre las dos posiciones.

2) Podemos considerar la realidad: a) como algo abstracto (up there), un formalismo estricto en el campo de esencias abstractas, como un producto o una sustancia estática, b) como algo concreto (down there), un empiricismo estricto en el campo de realidades concretas, como un proceso o acción dinámico, c) o como algo entre las dos posiciones.

\footnotetext{
${ }^{17}$ La retórica epistémica, o la retórica como manera de conocer (way of knowing) es el enfoque americano, contemporáneo, que sostiene que la producción del saber es una de las funciones importantes de la retórica. $Y$ en este movimiento epistémico se pueden distinguir dos líneas de investigación: a) la perspectiva dramatista que centra la retórica sobre los valores, b) y la perspectiva ideológica, que enlaza la retórica con la crítica social.
} 
Estos ejes pueden representar diferentes procesos cognitivos para capturar la realidad y la experiencia, y diferentes dimensiones o potencialidades. Pero entendemos que la cuestión que más nos importa aquí son sus relaciones dialécticas y los resultados en el discurso. Estas relaciones dialécticas convergen en un contexto argumentativo, en unas maneras de ver el mundo que sustenta todo discurso, y las diferentes maneras de usar la lengua. La lengua tiene un rol central y una preeminencia en la construcción de un mundo social, y sobre todo en la construcción de una acción dentro de este mundo social. Ello hace que la construcción del discurso no sea sólo una cuestión de un proceso cognitivo individual, sino que también es un proceso cognitivo social.

Para ambas realidades, la interior y la exterior, utilizamos las palabras para obtener una idea más o menos verdadera de ellas. La otra verdad, es la verdad del uso de la lengua que construye esa realidad. Pues, es, según la hipótesis de SapirWorf $^{18}$, la estructura del vocabulario y de la gramática de la lengua de un individuo la que realmente da forma a su visión del mundo. Esta hipótesis muestra cómo la lengua es una guía de la realidad social, y cómo el mundo real es, en gran parte, inconscientemente construido sobre los hábitos lingüísticos de la comunidad. Ellos muestran cómo las sociedades están a merced de sus lenguas, y cómo hablantes de dos lenguas diferentes viven dos mundos distintos.

Con todo ello, podemos afirmar que:

a) En la construcción de la realidad interviene la lengua a través de unos procesos cognitivos y mediante una acción que está asociada a unas estructuras lingüísticas, las cuales se despliegan en un contexto argumentativo. Este contexto hace que el discurso sea construido, no por un solo individuo, sino por dos o más participantes actuando.

b) El intercambio de la lengua en una determinada situación constituye y construye, sobre una adecuación comunicativa ${ }^{19}$, una realidad comunicativa. Así, el texto publicitario anterior es un ejemplo de cómo sus simples y breves estructuras lingüísticas construyen una realidad comunicativa, dentro de un marco retórico y un proceso argumentativo (que por otra parte caracteriza al discurso publicitario):

[No matter what your body type, this is the best workout video for you] $\hookrightarrow$ (a través de un proceso de inferencia regresivo, conduce a una conclusión que tiene una posición inicial en el texto) you can $\hookrightarrow$ [LOOK GOOD. FEEL BETTER. BE YOUR BEST].

La realidad, prácticamente, consiste en una concepción del significado. Y a través de la lengua encontramos, para esta concepción, términos, estructuras,

\footnotetext{
${ }^{18}$ McCormack, (1977:4) resalta que la hipótesis de Sapir-Worf, actualmente, no está ni completamente aceptada, ni completamente rechazada.

${ }^{19}$ El término "communicative adequacy" fue introducido por Hymes (1972); y adoptado, más tarde por Dik (1978) como un objetivo metodológico de la Gramática Funcional.
} 
textos, y discursos que son alternativos para presentar diferentes modelos de realidad. Una realidad que es relativa a los diferentes usuarios de la lengua y a las diferentes situaciones. Y según esto, ¿podríamos hablar de una realidad semántica, y de una realidad pragmática?. Ya hemos visto que la semántica está sistemáticamente interrelacionada con la pragmática. Pero desde la lógica construcción del significado, podemos tener en cuenta la relación establecida por Leech (1980:82):

A

Logical sense logico-pragmatic mapping
$B$

Pragmatic Force

Aquí, la semántica (Logical sense) parece ser previa a la pragmática (Pragmatic Force) en esta construcción lógica del significado. Leech contrasta esta relación con la establecida por Searle (1975) para con los actos indirectos de habla, donde el acto ilocutivo es realizado indirectamente por medio de otro. Así, este contraste se refleja en la siguiente relación (Leech, 1980:83):

A

Direct Illocution
$B$

Indirect Illocution

Desde este contraste, nosotros entendemos que hay una confluencia simultánea entre la estructura lógica o profunda (Logical sense) y la fuerza pragmática; y que es la operación lógico-pragmática, la que es previa (en la construcción lógica del significado) a un sentido semántico y a una fuerza pragmática. Entendemos por operación lógico-pragmática la realización de los conceptos de buena-construcción y buena-adecuación en el texto, y de acuerdo con la situación comunicativa. Ello conduce a la construcción y sostenimiento de una realidad comunicativa, donde, en la construcción lógica del significado, entendemos que operan tanto una lógica semántica como una lógica pragmática, relacionadas respectivamente con los conceptos anteriores. Nuestra propuesta quedaría esquematizada de la siguiente manera:

Trazado lógico

Buena construcción $\hookrightarrow$ Sentido Lógico

REALIDAD

pragmático

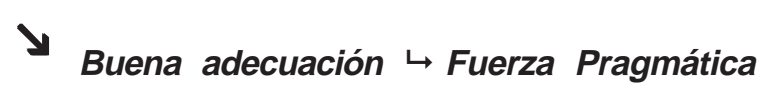


Los conceptos de buena-construcción y buena-adecuación llevan consigo una gran variedad de alternativas en su realización; pero también puede que esta realización se oriente hacia "mala-construcción / mala-adecuación". Por ejemplo, un texto con expresiones elípticas requerirá unas conexiones lógicas implícitas para entender el mensaje, y ello le daría una mayor fuerza pragmática según la situación comunicativa. Pero si este entendimiento no se logra, puede que haya una orientación y construcción inapropiada.

La lógica puede ser concebida como el conjunto de reglas que gobiernan y determinan las "exitosas" secuencias textuales del discurso (Kinneavy, 1971:64,107). Y aquí, por "existosas" entendemos buena-construcción y buena-adecuación. Donde la gramática viene a ser, en la práctica, una lógica sintáctica (Fish, 1989:478). Las reglas sintácticas son lógicas o tienen un carácter lógico para así llegar a construir estructuras que logren la comunicación (buena-construcción). Los principios retóricos se mueven en una lógica para hacer la comunicación más efectiva (buena-adecuación). Pero nosotros estructuramos el mundo a través de unas estructuras sintácticas que son una representación del significado semántico. Así, el sentido (sense) lógico de un texto, o de una proposición, viene determinado por el contenido que se expresa en él, y por las formas y modos gramaticales que lo construyen. Estas estructuras sintácticas llevan consigo una estructuración lógica que emerge de la dinámica racional en la interacción comunicativa, donde la lógica situacional y cultural marca la situación comunicativa y su perspectiva funcional en el texto, y establece una correspondencia entre la estructura del texto y la estructura del mundo.

Así, el siguiente texto de una emisión política (del grupo político Labour Party) en la televisión Británica antes de las elecciones:

\section{COMMENTATOR:}

What will happen if Labour win the next election? What kind of country will we live in? TONY BLAIR:

Tonight I'll try and answer those questions. But at the end of this broadcast if you still have more questions to ask l'll give you a phone number to call and ask me directly I care about what's happening to this country, about increasing job insecurity, about the rising crime rate, about falling standards of education for our children, about our National Health Service. I know you care about those things too. And tonight I intend to answer hard questions about what Labour will do about them in government. We're not promising the earth; we are promising what we can deliver. More jobs, more nurses, smaller classes, safer streets, success in business -these are the issues that matter to you. They're the issues that matter to me, too. Mine is the first generation to grow up not knowing if our children will be better off than we are; the first generation to fear for the improvement of our children's education and for our parents' care in old age. It shouldn't be like this: Britain is a great country, it is full of talented people, capable of competing with anyone in the world. Our potential is limitless, yet because the Conservatives only act for and care about a few people at the top, we're falling behind other countries. Our pledge to you is simple: new Labour will give new life to Britain. You'll find our plans in this document, NEW LABOUR, NEW LIFE FOR BRITAIN, which you can get by calling 
the number at the end of the broadcast. My colleagues and I will then be going out and about in Britain to discuss these ideas with you. I want to know what you think, I want you to know what kind of people we are, what kind of government we'd be, I would also like to give you one of these cards, it spells out these early pledges that we're making tonight. Keep it, so that you know what you are voting for and if we, win keep it to make sure we deliver on what we promise. I promise you now that we will deliver We have changed the Labour Party, now the Labour Party wants to earn your trust to change Britain, New Labour, New Life for Britain. Your country my country Together we can build a new Britain.

muestra como, ante los televidentes británicos, el locutor pregunta (utilizando una estructura modal y no remota con will $^{20}$, y la conjunción subordinante if; y expresando con todo ello una probable realidad), por una realidad comunicativa a Tony Blair:

- What will happen if Labour win the next election? What kind of country will we live in?

$Y$, en su respuesta discursiva, Tony Blair construye una realidad lingüísticamente well-formedness y well-appropriateness, basada principalmente en las siguientes estructuras:

a) Una referencia, o llamamiento (retórico), a su auditorio para que éste pueda intervenir en esa realidad que él, y su partido, están construyendo. Las estructuras son, otra vez, modales y no remotas con will; expresando con ello la intención y la voluntad del hablante de que esta intervención ocurra:

- l'll give you a phone number to call and ask me directly / My colleagues and I will then be going out and about in Britain to discuss these ideas with you.

b) Expresando su/s intención/es y voluntad/es mediante estructuras modales con: el verbo promise ${ }^{21}$, y los modales auxiliares can, will. $Y$ dando al auditorio la decisión acerca de que ocurra la realidad comunicativa que están construyendo:

- we are promising what we can deliver / to make sure we deliver on what we promise / I promise you now that we will deliver.

c) Construyendo una realidad comunicativa mediante dos estructuras paralelas de enumeración de hechos, en una relación de contraste. Encabezadas por la realización de los actos ilocutivos que los verbos care about y promise realizan en un modo declarativo. $Y$ acabadas, también, por unas estructuras paralelas, donde

\footnotetext{
${ }^{20}$ "Will has traditionally been consisted as a marker of future tense. However, will is not an auxiliary related to tense, but to mood. There is no future tense in English. The difference between the verb forms will see and see is not of tense but of mood: will see is non-remote and modal, see non-remote and non-modal' (Quereda, 1993:74).

${ }^{21}$ Mediante este verbo, además de expresar el significado modal de la actitud del hablante hacia lo que está diciendo, se realiza el acto ilocutivo, específico, de prometer.
} 
el elemento más relevante es el pronombre personal you, cuyo uso deíctico añade una fuerza retórica interpersonal al discurso.

- I care about what's happening to this country, about increasing job insecurity, about the rising crime rate, about falling standards of education for our children, about our National Health Service. I know you care about those things too.

- We're not promising the earth: we are promising what we can deliver. More jobs, more nurses, smaller classes, safer streets, success in business -these are the issues that matter to you.

Entendemos que estas estructuras que construyen la realidad, la del texto y la del mundo, funcionan, retórica y argumentativamente, desde el discurso. Y éste, el discurso, es esencialmente, tal como lo plantea Edward (1997:23): lengua, representación mental, y comunicación.

\section{DISCURSO: CONSTRUCCIÓN Y SUSTENTACIÓN}

Condor y Antaki (1997) hacen un estudio sobre la cognición social y el discurso. Por cognición social se entiende el conocimiento que la gente tiene del mundo social en el cual vive, habla y actúa. En este estudio de cognición social, ellos se centran en dos sentidos:

A) Uno orientado hacia los mecanismos sicológicos por el cual los individuos representan objetos sociales, y se perciben ellos mismos y a los demás en unos modos y circunstancias particulares. Se trata del procesamiento mental de la información acerca del mundo social (Mentalist Social Cognition).

B) Otro orientado a la naturaleza social de los perceptores y al mundo social que ellos construyen. Se trata de la construcción social de nuestro conocimiento acerca del mundo (Social Basis of Cognition).

Estos dos sentidos tienen diferentes aplicaciones al discurso:

A) "El procesamiento mental de la información acerca del mundo social" ofrece dos dominios: la clasificación del mundo que nos rodea a través de la categorización y unos esquemas y modelos; y la combinación y el cálculo de la información que estas categorías proporcionan: atribución de causa, actitudes, e inferencia social.

- La categorización es considerada como un rasgo básico de los procesos mentales humanos, mediante los cuales, para simplificar la tarea de percibir y reaccionar a los estímulos que encontramos en el mundo tendemos a utilizar categorías generales (perros, muebles, gente que nos gusta, etc.). Estas categorías son estructuras mentales que nos proporcionan, de forma automática, inferencias que guían nuestras acciones. La relevancia de esta categorización con respecto al discurso está en que ello podría explicar por qué dividimos el mundo originando unas categorías discursivas (gatos, pilotos, países) y no otras (por ejemplo: gente delgada que ha ido recientemente al dentista), y en cómo esta limitación afecta, posteriormente, a nuestras prácticas discursivas. 
- Los esquemas y modelos presuponen que la gente guarda mentalmente planes bien articulados de situaciones rutinarias y sus conductas correspondientes en ellas. Estos planes son, también, formas de construir el mundo social, disponiéndonos a tener una realidad construida de esta forma. Esto tiene que ver con ciertos aspectos discursivos esquematizados (las maneras de narrar y recordar historias, o el éxito o el fracaso de los chistes, ejemplos tratados como buenos o como cuestionables, etc.), y con prácticas discursivas como la formación de estereotipos y la discriminación por razón de género, raza, etc.

- La atribución de causa se refiere al proceso mental por medio del cual tratamos de explicar los eventos que abordamos en nuestras vidas. Tomamos información del evento y elaboramos, racionalmente, cuál es la causa probable. Al igual que en los procesos involucrados en la categorización, estos procesos mentales luchan con un mundo rudimentario o incompleto y lo canalizan en algo con lo que podemos tratar o manejar. Esto proporciona al discurso un mecanismo determinista que explica por qué la gente llega a ciertas explicaciones y procesamientos informativos. Para nosotros este proceso mental intervendría fundamentalmente en las estructuras concluyentes de la lengua, y acerca de la realidad. En el ejemplo anterior, del texto de la emisión política, la estructura: "I care about what's happening to this country, about increasing job insecurity, about the rising crime rate, about falling standards of education for our children, about our National Health Service", conduce mentalmente el discurso a las siguientes estructuras concluyentes, y a su utilización apropiada para constuir la realidad que se quiere comunicar: Our pledge to you is simple: "new Labour will give new life to Britain / Together we can build a new Britain".

- Las actitudes son un proceso evaluativo mental que se generan a través de tres componentes: "creencias y sentimientos causando acción". Las actitudes hacia el cambio han implicado el estudio de los efectos de los mensajes en las creencias y sentimientos de la gente, y de si, consecuentemente, su conducta exterior cambia. Entendemos que esto implicaría la relevancia de los discursos con unos propósitos de creencia y evaluación por parte del emisor, y la relevancia de la conducta lingüística del receptor como respuesta a lo anterior.

- La inferencia social es una facultad que trabaja sobre la información que entra al sistema cognitivo para sacar conclusiones acerca de la gente y los eventos. Es más bien como trabajar sobre la categorización. Nosotros creemos que a este proceso de inferir le son imprescindibles las estructuras lingüísticas que son utilizadas en las interacciones comunicativas. Pues necesitamos sacar conclusiones, y para ello necesitamos estas estructuras lingüísticas.

En definitiva, "el procesamiento mental de la información acerca del mundo social" se preocupa de la operación del procesamiento de la información universal, automática e inconsciente en la producción y comprensión del texto y del discurso. 
B) "La construcción social de nuestro conocimiento acerca del mundo" considera al conocimiento humano como un producto social bajo una propiedad compartida. Aquí, Condor y Antaki agrupan todos estos enfoques en tres amplias perspectivas $^{22}$ : el Conocimiento como Conducta Cultural (The Cognizer as Cultural Conduit), el Conocimiento como Miembro de un Grupo (The Cognizer as Group Member), la Cognición en el Intercambio Interpersonal (Cognition in Interpersonal Exchange).

1. El Conocimiento como Conducta Cultural. Considera a los individuos como portadores (y manipuladores) de una cultura particular, o un conjunto de ideologías compartidas. Aquí, Billig (1991) hace su enfoque con una clara relación al discurso, interesándose por el pensamiento, la retórica y la ideología. Él considera las creencias y percepciones de los individuos como una cuestión de ideología más que como un mero fenómeno cognitivo. De esta forma, Billig ve al perceptor social involucrado en un diálogo interno, en el cual lucha para dar sentido al mundo, y utilizando presunciones contradictorias y sentidos comunes que son proporcionados por su cultura. Es decir, el discurso y los contenidos de nuestro conocimiento social son cuestiones de un bagaje cultural e ideológico compartido. Él sugiere que un conocimiento del discurso humano y, en particular, de las técnicas de retórica, nos pueden informar de la naturaleza del pensamiento humano. $Y$ argumenta que la gente no sostiene una explicación de un fenómeno o evento dado, sino que más bien ellos son conscientes de y barajan concurrentes explicaciones.

2. El Conocimiento como Miembro de un Grupo. Considera a los individuos como miembros de distintos grupos, con intereses particulares compartidos. Aquí se enfatiza la relevancia de pertenecer a un grupo específico para la acción y cognición individual, incluyendo los actos discursivos. El actor social habla y piensa como parte de, en nombre de, una identidad colectiva. La acción y la percepción social son, en ocasiones, determinadas por la tendencia de los individuos a interiorizar las necesidades e intereses de los grupos específicos con los que ellos se identifican. En este sentido, ha habido poco análisis directo de los posibles puntos de intersección entre la identidad social como proceso cognitivo y acción social y las perspectivas analíticas del discurso. Utilizando la lengua natural como corpus, Reicher (1991) ha analizado la manera en la cual la prensa Británica construyó unas categorías sociales durante la guerra del Golfo. Entre otras, Reicher analiza la forma en que Saddam Hussein es utilizado para representar metonímicamente a Iraq, y las formas en las cuales Iraq fue aludido metafóricamente como una persona con atributos individuales.

3. La Cognición en el Intercambio Interpersonal. Se centra en los procesos de intercambio personal, los cuales pueden implicar la manera sobre la cual la realidad social puede ser conjuntamente construida. La cognición es parte esencial de la

\footnotetext{
${ }^{22}$ "This classification system is only a rouge typology, and it cannot capture the complexities of, and contradictions between, the many approaches which treat cognition as socially shared or as a social product." (Condor y Antaki, 1997:329).
} 
acción y de la interacción, y aquí se une con la lengua, teniendo ésta un papel central en estos procesos de interacción. Tradiciones como el interaccionismo simbólico de Mead $^{23}$ y la filosofía lingüística anglófona siempre han insistido en la primacía de la lengua y su rol en construir el mundo social y la acción dentro de él. Otras teorías, como la hipótesis de Sapir-Whorf, subrayan la importancia de la lengua en suministrar a la mente categorías que construyen, a su vez, el mundo de los objetos. En todo caso, desde esta perspectiva de intercambio personal, la cognición está vinculada a la acción, constituyendo así una realidad social. En esta vinculación, la lengua tiene una acción pública, y es el medio fundamental para constituir la realidad social. De esta forma, el discurso es una cuestión pública construida por muchas manos, y sirviendo a unos fines institucionales y locales. Y en esta perspectiva, Edwards y Potter (1992) ${ }^{24}$ resaltan la primacía de la lengua como una parte constitucional de la vida social, en la emergencia de la realidad social a través del intercambio entre los hablantes de una sociedad. Para ellos, la interacción interpersonal a través de la lengua es crucial para los procesos sociales. Por otro lado, Bartlett (1958) plantea la acción de pensar dentro de un contexto social, el cual es socialmente responsable de esa acción. En ello, Psathas (1978:132) ve que se pueden descubrir los métodos que las personas usan en su vida social diaria construyendo la realidad social, y también se puede descubrir la naturaleza de las realidades que ellos han construido. Aquí, nosotros entendemos que es el contexto social el responsable de la construcción que la lengua y la acción de pensar hacen de la realidad. Por ello no podemos ver la lengua sin su contexto de uso:

\begin{abstract}
...we shall have to attend to the words used as exactly as possible to see what might be going on: all the literary, stylistic and persuasive devices which speakers use to win over their listeners, with or without recourse to the hard coupling of formalizable premise and conclusion (Condor y Antaki, 1997:337).
\end{abstract}

En esta consideración de que el intercambio de la lengua constituye la realidad hay otros tipos de análisis como el del filósofo G. H. Mead y el del filólogo Bajtín. Para ambos, la proposición y la cognición carecerían de sentido sin la apreciación de su autoría conjunta o múltiple por parte de los hablantes. Para Mead se trata de una confabulación entre hablante y auditorio. Para Bajtín se trata de la infiltración de los intereses y perspectivas de un hablante en las proposiciones o enunciados del otro hablante.

\footnotetext{
${ }^{23}$ "Mead (1934): a philosophical knowing statement of the place of cognition in the social world" (Condor y Antaki, 1997:344).

${ }^{24}$ "Potter and Wetherell's Discourse and Social Psychology (1987) was a landmark in the application of anglophone discursive thinking to social phenomena and has recently been extended by Edwards and Potter's Discursive Psychology (1992) with a remit ranking over the wider spectrum of cognitive processes" (Condor y Antaki, 1997:335).
} 
Por otro lado, dentro del Análisis de la Conversación (CA) y la Etnometodología, Sacks $(1992)^{25}$ muestra como las palabras de la gente proponen y disponen acciones de forma diferente en diferentes puntos de una interacción. Por ejemplo, la palabra "Hello" tiene una fuerza diferente cuando es empleada al principio de una conversación (por teléfono), en un saludo, o como medio de identificar al hablante, que cuando es empleada en mitad de la conversación para comprobar que el otro interlocutor está ahí todavía. Así, lo que un hablante propone, será dispuesto por el otro hablante, y de esta forma ambos explotan las complejas regularidades de la interacción para hacerse con el significado. Derek Edwards sigue la línea etnometodológica para tratar los objetos mentales como cosas cuya realidad es la invocación que se hace de ellos en los diferentes tipos de conversaciones: de trabajo, íntima, fortuita o informal, científica. En Edwards (1991) muestra como las declaradas universalidades de la "categorización" no son para ser consideradas como verdad, sino como parte de su carga (útil) retórica. En Edwards (1994) trata la noción de "scripts" como reglas culturales que pueden ser invocadas o explotadas en situaciones apropiadas.

En definitiva, "la construcción social de nuestro conocimiento acerca del mundo" considera que la cognición está unida a la acción conjunta de la gente, y se preocupa del discurso como una acción social que es conjuntamente ocasionado y formulado sirviendo a unos fines locales e institucionales.

Pero el discurso tiene que ver, además de con la naturaleza del mundo exterior, con la naturaleza del mundo interior. Es decir, con la realidad y con la mente, y con las relaciones que hay entre las dos. Dos naturalezas, donde media la naturaleza de la lengua, en un sistema de sonidos, palabras, gramática y retórica.

$\mathrm{Y}$, sobre un esquema discursivo, pueden contemplarse dos procesos de acción del discurso por parte del hablante: a) un proceso de elección y construcción sobre la relación discurso-realidad exterior, b) y un proceso complementario que construye, mediante el discurso, una realidad interna sustitutiva de esa realidad exterior. Todo ello lo representamos de la siguiente manera:

\footnotetext{
25 "Sacks (1992): edited transcripts of Sacks's lectures from the 1960s and 1970s; a fascinating and stimulating store of insights into human action, putting the argument that it is to be understood without risking the epistemological dubiousness of mental entities." (Condor y Antaki, 1997:344).
} 


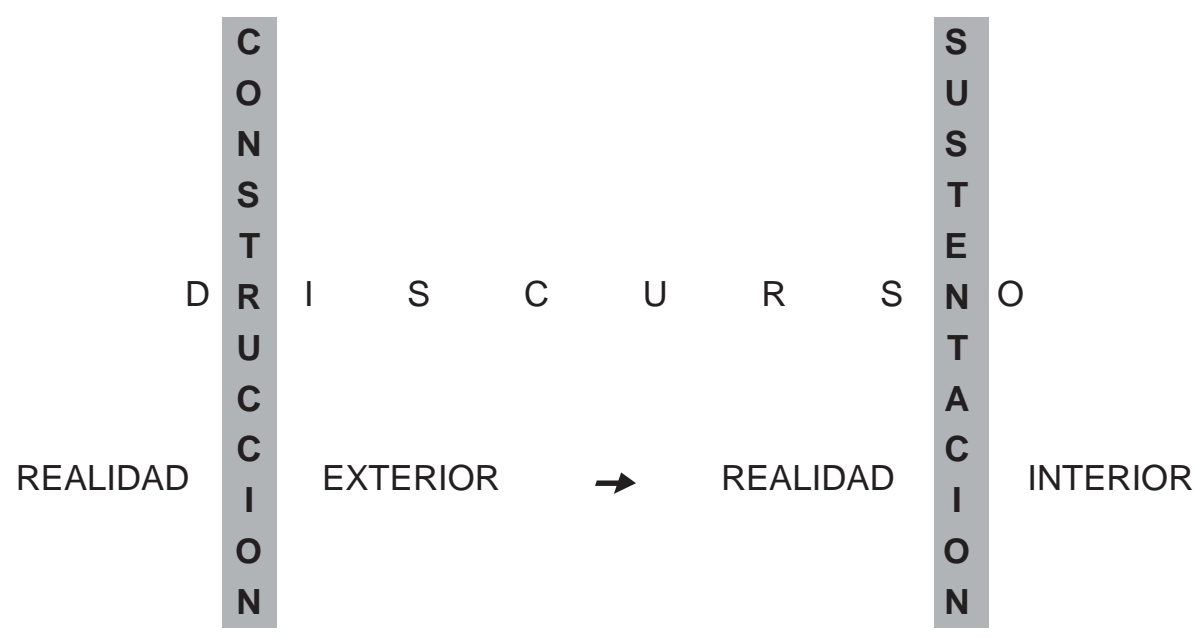

El discurso desarrolla una acción retórica, al constituirse sintáctica y semánticamente, y al proyectarse pragmáticamente desde el hablante al auditorio. Esta acción retórica construye una realidad exterior en la comunidad lingüística determinada, y sustenta una realidad interior de creencias y convicciones en los participantes de la interacción comunicativa ${ }^{26}$. Todo ello sobre la base de cómo funciona la lengua:

The way language works, then, is that each person's brain contains a lexicon of words and the concepts they stand for (a mental dictionary) and a set of rules that combine the words to convey relationships among concepts (a mental grammar) (Pinker, 1994:85).

Ello nos conduce al texto y a sus estructuras, donde la realidad se construye y sustenta, a través de lo que Jackendoff (1987) llama la "estructura lógica del procesamiento de la lengua":

....given the organization of language, one cannot accomplish Esther speech understanding or speech production without making essential use of both phonological and syntactic information. I will codify this as the Logical Structure of Language Processing (Jackendoff, 1987:92).

\footnotetext{
${ }^{26}$ Esta relación entre realidad exterior y realidad interior, construida y sustentada, respectivamente, por el discurso, podría tener cierta proyección con la distinción, en psicología cognitiva, entre public language / private language. Ver Fodor (1979).
} 
Pero lo más notable de esta "estructura lógica del procesamiento de la lengua" es la carga de intencionalidad comunicativa que procesa en la interacción hablante/ oyente, y el carácter convencional que, por su uso cotidiano, puede establecer en sus estructuras y significados. Jackendoff (1987:128) sitúa la intencionalidad en esa relación relevante que hay entre los estados mentales y tal como el mundo es procesado. En la figura 2, esta intencionalidad y este carácter convencional se situarían en la relación relevante entre el discurso y la construcción/sustentación de las realidades. Es decir, en dos aspectos íntimamente relacionados: la elección o selección, por parte del hablante, de unas estructuras lógicas del procesamiento de la lengua, y el efecto estimulado en el auditorio, de acuerdo con el procesamiento de estas estructuras lógicas.

El texto anterior, de la emisión política, por ejemplo, muestra claramente como la intencionalidad de todo el discurso de Tony Blair está de forma implícita en todo el discurso; y de forma explícita en las siguientes estructuras, al final del texto:

-...now the Labour Party wants to earn your trust to change Britain, New Labour, New Life for Britain. Your country my country. Together we can build a new Britain.

De modo que, estas estructuras y significados, cargados de un modo declarativo [...wants to earn your trust], y de una modalidad de posibilidad [...we can build..], pueden esquematizar el discurso (o la realidad comunicativa) que Tony Blair construye y utiliza para crear una realidad exterior [Together we can build a new Britain], y sustentar una realidad interior [...your trust to change Britain]. Pero ambas realidades se basan en el texto que se construye y utiliza.

\section{CONCLUSIONES}

Dentro de cada discurso hay una racionalidad construida, a través de sus estructuras, y a través de un contenido que es lógicamente coherente. La referencia del texto obedece a alternativas lógicas particulares y sociales. Y siempre, en nuestro uso de la lengua, realizamos procesos de razonamiento. Esta racionalidad es adaptable a los interlocutores y a las situaciones, y es variable de acuerdo con las capacidades de los participantes en la comunicación y de acuerdo con los cálculos estratégicos.

Usar la lengua es interpretar la realidad, trazándola, al menos, desde dos perspectivas: la del hablante y la del oyente. Aunque, podrían contemplarse otras perspectivas en la interacción textual, si consideramos que pueden existir otras voces y otros auditorios implicados en esa interacción. Pero, la lengua no solamente construye la realidad, sino que además, ayuda a crear, y refleja, los intereses y las ideas de los grupos sociales dominantes. De modo que a través de la lengua:
a) se puede crear, modificar, y desarrollar un sentido del mundo,
b) se puede institucionalizar lo que es hablado frecuentemente, 
c) y se puede debilitar o percibir como inaceptable otras versiones del uso de la lengua que son omitidas.

Ello hace que la construcción del discurso no sea sólo una cuestión de un proceso cognitivo individual, sino que también es un proceso cognitivo social.

Con todo ello, podemos afirmar que en la construcción de la realidad interviene la lengua a través de unos procesos cognitivos y mediante una acción, que está asociada a unas estructuras lingüísticas, los cuales se despliegan en un contexto argumentativo, donde la construcción y el sostenimiento de una realidad comunicativa constituyen una operación lógico-pragmática, mediante la realización de los conceptos de buena construcción y buena adecuación en el texto, y de acuerdo con la situación comunicativa. Aquí, las reglas sintácticas son lógicas o tienen un carácter lógico para así llegar a construir estructuras que logren la comunicación (buena construcción). Y los principios retóricos se mueven en una lógica para hacer la comunicación más efectiva (buena adecuación). Por lo tanto, en la construcción lógica del significado operan tanto una lógica semántica como una lógica pragmática.

Pero el discurso tiene que ver, además de con la naturaleza del mundo exterior, con la naturaleza del mundo interior. Es decir, con la realidad y con la mente, y con las relaciones que hay entre las dos. Y, sobre un esquema discursivo, pueden contemplarse dos procesos de acción del discurso por parte del hablante:

a) un proceso de elección y construcción sobre la relación discurso-realidad exterior,

b) y un proceso complementario que construye, mediante el discurso, una realidad interna sustitutiva de esa realidad exterior.

Por lo tanto, el discurso desarrolla una acción retórica, al constituirse sintáctica y semánticamente, y al proyectarse pragmáticamente desde el hablante al auditorio. Y esta acción retórica construye una realidad exterior en la comunidad lingüística determinada, y sustenta una realidad interior de creencias y convenciones en los participantes de la interacción comunicativa.

\section{BIBLIOGRAFÍA:}

ANSCOMBRE, Jean-Claude 1991. "Dynamique du sens et scalarité". En: A. Lempereur, (ed.), L'Argumentation. Liège: Pierre Mardaga Éditeur, págs. 123-146.

BARLETT, F. C. 1958. Thinking. London: Unwin Hyman.

BAZERMAN, Charles y PARADIS, James (eds.) 1991. Textual Dynamics of the Professions. Historical and contemporary studies of writing in professional communities. Madison, Wisconsin: The University of Wisconsin Press. 
BEALE, Water H. 1987. A Pragmatic Theory of Rhetoric. Carbondale and Edwardsville, III.: Southern Illinois University Press.

BERGER, Peter L. y LUCKMANN, Thomas 1976. The Social Construction of Reality: A Treatise in the Sociology of Knowledge. Harmondsworth: Penguin Books.

BILLIG, Michael 1991. Ideology and Opinions: Studies in Rhetorical Psychology. London: SAGE Publications.

BORMAN, Ernest G. 1985. The Force of Fantasy: Restoring the American Dream. Carbondale, III.: Southern Illinois University Press.

BROWN, W. R. 1978. "Ideology as Communication Process", Quarterly Journal of Speech, 64: 123-140.

BURKE, Kenneth 1969. A Rhetoric of Motives. Berkeley/Los Angeles: University of California Press (publicado en 1950).

CONDOR, Susan y ANTAKI, Charles 1997. "Social Cognition and Discourse". En: T. A. van Dijk (ed.), Discourse as Structure and Process. Discourse Studies: A Multidisciplinary Introduction Volume 1. London: SAGE Publications, págs. 320-347.

DIK, Simon C. 1978. Functional Grammar. Amsterdam: North Holland.

Dik, Simon C. 1989. The Theory of Functional Grammar. Part I: The Structure of the Clause. Dodrecht: Foris Publications.

EDWARDS, Derek 1991. "Categories are for talking: on the cognitive and discursive bases of categorization", Theory and Psychology, no 1, págs. 515-42.

EDWARDS, Derek 1994. "Script formulations: an analysis of event descriptions in conversation", Journal of language and Social Psychology, № 13, págs. 211-47.

EDWARDS, Derek 1997. Discourse and Cognition. London: SAGE Publications EDWARDS, Derek y POTTER, Jonathan 1992. Discursive Psychology. London: SAGE Publications.

FAIRCLOUGH, Norman 1992. Discourse and Social Change. Cambridge: Polity Press.

FAIRCLOUGH, Norman 1995. Media Discourse. London, New York: Edward Arnold.

FAIRCLOUGH, Norman 2001. "The dialectics of discourse". Textus XIV (2): 231-242.

FISH, Stanley 1989. Doing what comes naturally. Change, rhetoric, and the practice of theory in literary and legal studies. Oxford: Clarendon Press.

FODOR, Jerry A. 1979. The Language of Thought. Cambridge, Mass.: Harvard University Press.

FOWLER, Roger 1996 ( $2^{\text {nd }}$ edition / 1a ed.:1986). Linguistic Criticism. Oxford/New York: Oxford University Press.

GRIZE, Jean-Blaise. 1982. De la Logique à L'Argumentation. Genève: Librairie Droz.

HALLIDAY, Michael A.K. 1978. Language as a Social Semiotic. The Social Interpretation of Language and Meaning. London: Edward Arnold. 
HYMES, Dell H. 1972. "Models of the Interaction of Language and Social Life". En: J. J. Gumperz y D. Hymes (eds.), Directions in Sociolinguistics. The Ethnography of Communication. New York: Holt, Rinehart and Winston, págs. 35-71 (También en 1986 edición).

JACKENDOFF, Ray 1987. Consciousness and the Computational Mind. Cambridge, Mass. - London: The MIT Press.

KINNEAVY, James L. 1971 (publicación por Norton: 1980). A Theory of Discourse. New York, London: Norton \& Company.

KRESS, Joseph 1992. Psicolinguistics: psychology, linguistics, and the study of natural Language. Amsterdam: John Benjamins.

LEECH, Geoffrey N. 1980. Explorations in semantics and pragmatics. Amsterdam: John Benjamins.

LEITH, Dick y MYERSON, George 1989. The Power of Address. Explorations in rhetoric. London, New York: Routledge.

MCCORMACK, William 1977. "Introduction". En: W. McCormack y S. Wurm (eds.), Language and Thought: Anthropological Issues. The Hague: Mouton Publishers, págs. 3-10.

MCGEE, Michael C. 1980. "The 'Ideograph': A Link Between Rhetoric and Ideology", Quarterly Journal of Speech no 66, págs. 1-16.

MEIJS, Willem 1992. "Inferences and lexical relations". En: G. Leitner (ed.) 1992. New Directions in English Language Corpora. Methodology, Results, Software Developments. Berlin, New York: Mouton de Gruyter, págs. 123-142.

O'KEEFE, Barbara J. 1986. "The Functional Integration of Communication Concepts: Evidence for Individual Differences in Reasoning About Communication". Communicacion en: "Annual Meeting of the Speech Communication Association", Chicago, Illinois.

OWEN, James L. (ed.) 1997. Context and Communication Behaviour. Reno (Nevada): Context Press.

PERELMAN, Chaïm 1983. "Logique formelle et argumentation". En: P. Bange, A. Bannour, A. Berrendonner, O. Ducrot, C. Kohler-Chesny, G. Lüdi, Ch. Perelman, B. Py, E. Roulet, (eds.), Logique, argumentation, conversation: Actes du Colloque de Pragmatique, Fribourg, 1981. Berne: Peter Lang.

PINKER, Steven 1994. The Language Instinct. How the Mind Creates Language. New York: William Morrow and Company

PLANTIN, Christian 1990. Essais sur l'Argumentation. Introduction linguistique à l'étude de la parole argumentative. Paris: Kimé.

POTTER, Jonathan y WETHERELL, Margie 1987. Discourse and Social Psychology: Beyond Attitudes and Behaviour. London: SAGE Publications.

PSATHAS, George 1978 (3ㄹ ed.). "Ethnomethods and phenomenology". En: J. G. Manis y B. N. Meltzer (eds.), Symbolic Interaction: a Reader in Social Psychology. Boston: Allyn and Bacon. 
QUEREDA RODRÍGUEZ-NAVARRO, L. 1993. A Morphosyntactic Study of the English Verb Phrase. Granada: Universidad de Granada.

REICHER, S. 1991 "Mad dogs and Englishmen: telling tales from the Gulf", artículo presentado en The British Association 'Science 91' meeting, Plymouth.

SACKS, Harvey 1992. Lectures on Conversation (ed. Gail Jefferson). Oxford: Basil Blackwell.

SAYER, Andrew 2000. Realism and the Social Sciences. London: SAGE Publications.

SEARLE, John R. 1995. The Construction of Social Reality. London: Penguin Books.

TOULMIN, Stephen 1983. "Logic and the Criticism of Arguments". En: J. Golden, G.

Bergquist y W. Coleman, The Rhetoric of Western Thought, Dubuque, lowa: Kendall/ Hunt, págs. 391-401.

WHORF, Benjamin Lee 1956. Language, Thought, and Reality: Selected Writitngs of Benjamin Lee Whorf. Editado e introducido por J. B. Carroll. Cambridge, Mass.: M.I.T. Press.

WILLARD, Charles A. 1991. "L'argumentation et les fondements sociaux de la connaissance”. En: A. Lempereur (ed.), L'Argumentation. Liège: Pierre Mardaga Éditeur, págs. 91-106.

(Recibido el 11-11-05, aceptado el 25-02-06) 